\title{
Numerical simulation of Energetic materials burning based on material point method
}

\author{
Wei-dong Chen ${ }^{\mathrm{a}}$, Feng-chao Zhang ${ }^{\mathrm{b}}$, Sheng-zhuo $\mathrm{Lu}^{\mathrm{c}}$ \\ College of Aerospace and Civil Engineering Harbin Engineering University, Harbin 150001 \\ Heilongjiang, China

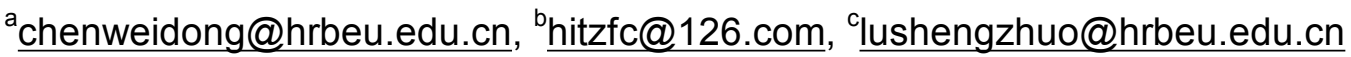

\begin{abstract}
Keywords: Material point method; Energetic materials Combustion; Numerical Simulation; Closed Bomb; Combustion characteristics.

Abstract. Numerical simulation of Energetic materials combustion and Energetic materials burning rate determination is the key issues of Energetic materials combustion study. Material point method inherits the advantages of Lagrange and Euler, which have a great advantage when dealing with large deformation and multi-media coupling problem. On the basis of the material point method, research energetic materials combustion problems, establish energetic materials combustion model based on material point method, extend the material point method to the combustion numerical computing. With the numerical simulation of the closed bomb test, get the pressure-time curve, burning rate-pressure curve and others combustion characteristic curve of the measuring point in energetic materials, which were in good agreement compared with experimental data and burning rate - pressure formula. Numerical simulation of energetic materials burning based on material point method provides a new way to the energetic materials combustion simulation and provides the numerical method and an important reference to study the combustion characteristics of energetic materials..
\end{abstract}

\section{Introduction}

In the research and development of new energetic materials, new combustion phenomena are increasing, especially in different pressure ranges and different dynamic environments, which may exhibit different combustion characteristics. With various new charging techniques and the development of weapons and equipment, energetic materials application environment has also undergone great changes, weapons and equipment emission process abnormal phenomenon gradually increased $^{[1]}$. As the most important method to test the combustion performance of energetic materials, the test results have played an important role in the research and application of energetic materials. The use of closed burst test to test the combustion of combustion drugs in a closed environment when burning performance, is conducive to charge the designer to understand the actual combustion characteristics of the charge, and in accordance with the requirements of the ballot design to determine the parameters of the charge. However, when the closed-burst test tests the combustion performance of the energetic materials, the noise generated by the piezo-pressure measurement signal will mask the local combustion characteristics of the energetic materials. The heat loss will affect the accuracy of the test results. Accurate description of the energetic materials burning pressure index with the pressure of the characterization method ${ }^{[2-3]}$. The combustion performance of the energetic materials is not only an essential issue that restricts the effectiveness of weapons and equipment, but also a fundamental issue involving the safe and reliable application of energetic materials. Therefore, the simulation of the combustion process and the characteristics of the experiment can complement the experimental shortcomings, has a very important theoretical and practical significance.

In the analysis of explosions, shocks and other issues, the research method is generally numerical simulation. For the traditional numerical simulation method, the description of the physical control equation is generally divided into Lagrange and Euler. The Lagrange method is described by the coordinates of the material. The typical representation is the finite element method (FEM). The Euler method is described by spatial coordinates, which is typically represented by finite difference method (FDM). The lattice distortion is serious and can not be calculated even if the Lagrange method is used 
to deal with the large deformation problem ${ }^{[4]}$. The mesh of the Euler method is fixed in the calculation process and does not need to be meshed, There is no problem of grid distortion, but it can not track the field variables of the material in the calculation process. It is difficult to describe the interface of each material, and there is a convective term in the control equation, which will lead to complicated calculation.

In recent years, the biggest advantage of the rise of the meshless algorithm is that it can partially or completely eliminate the grid, so as to effectively avoid the numerical calculation caused by the dependence on the grid. Material point method (MPM) as a meshless particle algorithm, there is no grid distortion, there are convective items in the control equation, the field variable of the material is difficult to track and so on. For the impact penetration, explosion, Collision, fluid-solid coupling and other non-linear problems provide an effective method of analysis ${ }^{[5]}$. In this paper, the basic method of material point method is used to extend the material point method to the numerical field of combustion numerical calculation. The numerical simulation of the test process of the closed explosive device is carried out. The pressure time curve of the measuring point and the combustion rate-pressure curve of the energetic materials are obtained. The results show that the results are in good agreement with the experimental data and the burning rate - pressure formula. The results are in good agreement, which provides a new way for the combustion simulation of the energetic materials and provides a numerical method and important reference for the study of the combustion characteristics of the energetic materials.

\section{MPM Control Equation and Discretization}

In the process of material point method, the continuum must satisfy the conservation of mass and the conservation of momentum:

$$
\frac{d \rho}{d t}+\rho \nabla \cdot \boldsymbol{v}=0
$$

Where: $\rho=\rho(\boldsymbol{x}, t)$ for the density, $\boldsymbol{v}=\boldsymbol{v}(\boldsymbol{x}, t)$ the velocity vector, $\nabla \cdot \boldsymbol{v}$ the divergence of the velocity vector.

$$
\rho \frac{d v}{d t}=\nabla \cdot \sigma+\rho \boldsymbol{b}
$$

Where: $\boldsymbol{\sigma}=\boldsymbol{\sigma}(\boldsymbol{x}, t)$ for the stress tensor, $\boldsymbol{b}$ for the density of the force.

Since the mass and mass remain constant during the calculation process, the mass conservation equation is automatically satisfied and its density function is:

$$
\rho(\boldsymbol{x}, t)=\sum_{p=1}^{N_{P}} m_{p} \delta\left(\boldsymbol{x}-\boldsymbol{x}_{p}^{t}\right) .
$$

Where: $N_{P}$ for the total number of material points, $m_{P}$ the quality of the material point $p, \delta$ as a function of Dirac $\delta, \boldsymbol{x}_{p}^{t}$ the material point $p$ in the moment $t$ the location coordinates.

Multiply the weight function $\omega$ by (1-2), we obtain the equivalent integral of the momentum conservation equation.

$$
\int_{\Omega} \rho \boldsymbol{a} \cdot \omega d v+\int_{\Omega} \boldsymbol{\sigma}: \nabla \omega d v-\int_{\Omega} \rho \boldsymbol{b} \cdot \omega d v-\int_{\Gamma} \boldsymbol{\tau} \cdot \omega d s=0 .
$$

Where: $d v$ for the differential volume element, $d s$ for the differential area element, $\Gamma$ for the specified stress boundary, $\omega$ the specified displacement boundary is zero.

(1-3) into the formula (1-4), (1-4) of the integral form can be transformed into the following sum form: 


$$
\begin{aligned}
& \sum_{p=1}^{N_{P}} m_{p}\left[\omega\left(\boldsymbol{x}_{p}^{t}, t\right) \cdot \boldsymbol{a}\left(\boldsymbol{x}_{p}^{t}, t\right)+\frac{\boldsymbol{\sigma}\left(\boldsymbol{x}_{p}^{t}, t\right)}{\rho}: \nabla \omega\left(\boldsymbol{x}_{p}^{t}, t\right)\right] \\
& =\sum_{p=1}^{N_{P}} m_{p}\left[\boldsymbol{b}\left(\boldsymbol{x}_{p}^{t}, t\right) \cdot \omega\left(\boldsymbol{x}_{p}^{t}, t\right)+\frac{\omega\left(\boldsymbol{x}_{p}^{t}, t\right) \cdot \boldsymbol{\tau}\left(\boldsymbol{x}_{p}^{t}, t\right)}{\rho \cdot h}\right] .
\end{aligned}
$$

Where: $h$ for the boundary layer thickness.

In order to solve the gradient term in (1-5), we need to arrange the background mesh in the solution domain. The mapping relationship between grid nodes and material points is:

$$
\Phi_{p}^{t}=\sum_{i=1}^{N_{n}} \Phi_{i} N_{i}\left(\boldsymbol{x}_{p}^{t}\right) \text {. }
$$

Where: $\Phi_{p}^{t}$ for the grid node physical quantity, $\Phi_{i}$ physical point for the physical quantity, $N_{i}(\boldsymbol{x})$ for the node basis function, $N_{n}$ the total number of material points.

(1-4) can be written as a discrete form of grid nodes:

$$
m_{i}^{t} \boldsymbol{a}_{i}^{t}=\left(\boldsymbol{f}_{i}^{\mathrm{t}}\right)^{i n t}+\left(\boldsymbol{f}_{i}^{t}\right)^{e x t} .
$$

The quality of the nodes in the formula is:

$$
m_{i}^{t}=\sum_{p=1}^{N_{p}} m_{p} N_{i}\left(\boldsymbol{x}_{p}^{t}\right) .
$$

The internal force vector is:

$$
\left(\boldsymbol{f}_{i}^{\mathrm{t}}\right)^{\mathrm{int}}=-\sum_{p=1}^{N_{p}} m_{p} \boldsymbol{\sigma}\left(\boldsymbol{x}_{p}^{t}, t\right) /\left.\rho \cdot\left(\nabla N_{i}\right)\right|_{x=\boldsymbol{x}_{p}^{t}} .
$$

The external force vector is:

$$
\left(\boldsymbol{f}_{i}^{t}\right)^{e x t}=\sum_{p=1}^{N_{p}}\left[m_{p} \tau\left(\boldsymbol{x}_{p}^{t}\right) N_{i}\left(\boldsymbol{x}_{p}^{t}\right) /(\rho \cdot h)\right]+\sum_{p=1}^{N_{p}} m_{p} \boldsymbol{b}\left(\boldsymbol{x}_{p}^{t}, t\right) \cdot N_{i}\left(\boldsymbol{x}_{p}^{t}\right) .
$$

In summary, in the application of material point method to solve the actual mechanics problem, the continuum is separated into a certain quality of the particle $N_{P}$, and the background grid is covered in the solution domain. In the material point method, the mass carries all the information about the quality, speed, and the like, and indirectly through the background grid. In a time step, the particle information is mapped to the background mesh node, and the momentum of the node is updated by solving the momentum equation, and then the information such as the momentum carried on the node is mapped back to the particle. The information carried by the particle is updated by two mapping calculations. At the beginning of the next time step, the mapping process of the previous time is repeated until the specified time or the specified condition is reached, thereby completing the solution to the actual problem.

\section{Numerical Simulation of Combustion of Energetic Materials}

Constitutive equation of energetic material

For the mechanical response analysis of homogeneous energetic materials chemical reaction, the thermoelastic and plastic constitutive equation is used:

$$
\sigma_{\mathrm{ij}}^{\nabla}=C_{i j k l}\left(\dot{\varepsilon}_{\mathrm{kl}}-\dot{\varepsilon}_{\mathrm{kl}}^{(\mathrm{T})}\right)+\dot{\gamma}_{\mathrm{ij}} \mathrm{dT} \text {. }
$$

Where $\mathrm{T}$ the temperature of the propellant caused by the thermo-elastoplastic material of the propellant and the thermal decomposition of the thermal decomposition; $\dot{\varepsilon}_{\mathrm{kl}}$ is the total strain rate; $\dot{\varepsilon}_{\mathrm{k} 1}^{(\mathrm{T}}=\alpha \dot{\mathrm{T}} \delta_{i j}$ is the thermal strain rate; $\alpha$ is the linear expansion coefficient; $\delta_{i j}$ is the Kronecker symbol; $\dot{\gamma}_{\mathrm{ij}}=\frac{d C_{k l m n}}{d T} C_{k \operatorname{lmn}}{ }^{-1} \dot{\sigma}_{\mathrm{mm}}, C_{k \operatorname{lmn}}$ is the weight of the temperature-dependent elasticity the matrix $C$. The yield function of the material is: 


$$
\varphi=\frac{1}{2} S_{i j} s_{i j}-\frac{1}{3}\left[\sigma_{\mathrm{y}}(\mathrm{T})\right]^{2} \leq 0 .
$$

Where: $s_{i j}$ for the stress bias; $\sigma_{\mathrm{y}}(\mathrm{T})=\mathrm{E}_{\mathrm{p}}(\mathrm{T}) \varepsilon_{\mathrm{e}}{ }^{\mathrm{p}}+\sigma_{0}(\mathrm{~T})$ for the yield stress; $\varepsilon_{\mathrm{e}}{ }^{\mathrm{p}}$ for the equivalent plastic strain; $\sigma_{0}$ for the initial yield stress; $\mathrm{E}_{\mathrm{p}}$ for the plastic hardening modulus; $\sigma_{0}$ and $\mathrm{E}_{\mathrm{p}}$ are a function of temperature.

Thermal decomposition of energetic materials

For the energetic materials, the temperature rise will cause the thermal decomposition of the material heat release reaction, the chemical kinetics equation using Arrhenius equation. Since the conversion rate of the reactants is very low at the time of ignition, it is assumed that the initial concentration change of the reactants from the self-heating to the ignition is negligible. The thermal decomposition heat of the energetic materials is:

$$
Q_{t}=Q A \exp \left(-\frac{E_{a}}{R T}\right) .
$$

Where $\mathrm{Q}$ is the reaction heat, $\mathrm{A}$ is the pre-exponential factor, Ea is the activation energy, and $\mathrm{R}$ is the gas constant.

The state equation of energetic materials

Solid phase of the energetic materials using shock state equation:

$$
\begin{gathered}
p=p_{H}+\Gamma \rho\left(e-e_{H}\right) . \\
p_{H}=\frac{\rho_{0} c_{0}^{2} \mu(1+\mu)}{[1-(1-s) \mu]^{2}}, \quad e_{H}=\frac{1}{2} \frac{p_{H}}{\rho_{0}}\left(\frac{\mu}{1+\mu}\right) .
\end{gathered}
$$

Where: $\rho$ is the current density, $\rho_{0}$ is the initial density, $\mu$ is the degree of compression, $\mu=\frac{\rho}{\rho_{0}}-1, c_{0}$ and $s$ are the material parameters.

The gas phase product uses Abel equation of state:

The relationship between gas pressure and temperature:

$$
P_{g}=\frac{\rho_{g} R T_{g}}{1-b_{r} \rho_{g}} .
$$

The relationship between gas phase internal energy and temperature:

$$
e_{g}=C_{v g} T_{g} \text {. }
$$

Where: $R$ for the ideal gas constant, $C_{v g}$ for the gas phase capacity, $b_{r}$ for the gas capacity. among them,

$$
\begin{aligned}
b_{r}= & 1.8131 \times 10^{-3}-1.0326 \rho_{g} \times 10^{-6}+1.7722 \rho_{g}{ }^{2} \times 10^{-9} \\
& -2.0576 \rho_{g}{ }^{3} \times 10^{-12}+1.1472 \rho_{g}{ }^{4} \times 10^{-15} \\
& -0.2986 \rho_{g}{ }^{5} \times 10^{-18}+0.02945 \rho_{g}{ }^{6} \times 10^{-21}
\end{aligned}
$$

\section{Closed Burst Test}

The closed-burst test is a test to study the law of the pressure change during the combustion of the powder at the constant volume. The test method is used to detect and study the burning performance and the number of test bulletistics. It plays an important role in the static analysis of gunpowder, which is the main method to detect and study the burning performance of gunpowder. As early as the 960s, Nobel and Abel began to use the closed burst and copper pillar pressure measurement of gunpowder gas maximum pressure and the relationship between the charges. Today, the closed-burst test method has become the standard method for testing the combustion performance of gunpowder. The pressure measurement method also changes from the copper column pressure to the pressure sensor. The test 
parameters are also extended to the gunpowder, the residual volume, the law of burning rate The The structure of the diagram shown in Figure 1.

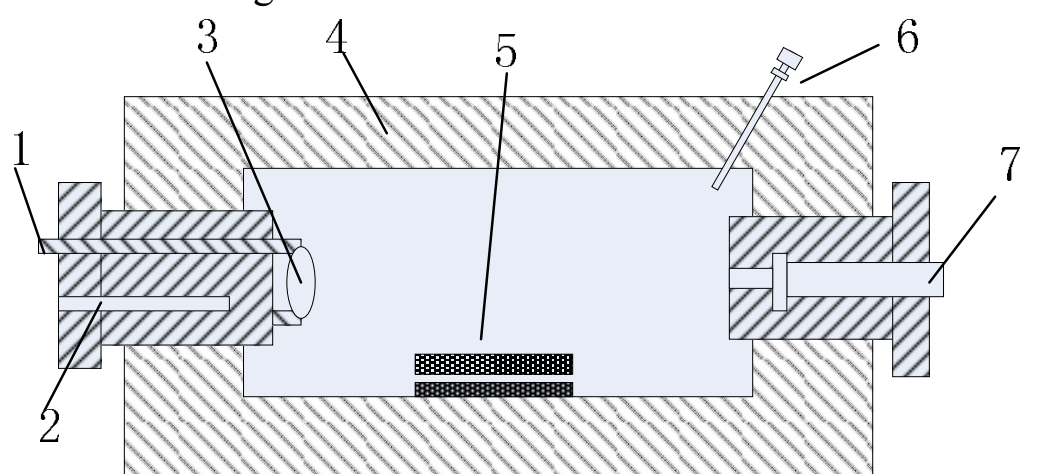

1-electrode, 2-escape hole, 3-igniting charge, 4-closed bomb, 5-propellants, 6-platinum resistance thermometer, 7-pressure measurement hole

Fig.1 Schematic diagram of Closed Bomb

The propellant used in this experiment was ${ }^{[6]}$ : bifang-3, Its parameters are: Nitrite $(26 \pm 10) \%$; dinitrotoluene $(9.0 \pm 1.0) \%$; dibutyl phthalate $(4.5 \pm 0.7) \% ; \pm 0.5) \%$; Vaseline: $(1.0 \pm 0.4) \%$.

The drug charge was tubular charge: drug length: $3.9920 \mathrm{~cm}$, flesh thickness: $0.1600 \mathrm{~cm}$, inner diameter: $0.2000 \mathrm{~cm}$, outer diameter: $0.5200 \mathrm{~cm}$, drug width: $0.3200 \mathrm{~cm}$, gunpowder density: 1.6000 $\mathrm{g} / \mathrm{cm}^{3}$.

Closed burst test according to GJB770B-2005, the use of high-pressure closed explosive body, the combustion chamber volume of $100 \mathrm{~cm}^{3}$, loading density: $0.2000 \mathrm{~g} / \mathrm{cm}^{3}$, ignition pressure: 10.000 Mpa.

\section{Numerical Examples and Result Analysis}

The numerical simulation of the experimental process is carried out using the plane model. The model is shown in Figure 2. Length $40 \mathrm{~mm}$, aperture $2.00 \mathrm{~mm}$, thickness $0.16 \mathrm{~mm}$. The enclosed burst has an internal diameter of $36 \mathrm{~mm}$ (volume $100 \mathrm{~m} 3$ ).

The propellant parameters were ${ }^{[7]}: \rho=1.83 \mathrm{~g} / \mathrm{cm}^{3}, c_{V}=1010 \mathrm{~J} /(\mathrm{kg} \cdot \mathrm{K}), A=5.9 \times 10^{14} \mathrm{~s}^{-1}$, $E_{a}=1.68 \mathrm{MJ} / \mathrm{mol}, Q=2.284 \mathrm{MJ} / \mathrm{kg}$, the failure strain was $0.66 \%$ and the ignition temperature was $615 \mathrm{~K}$.

The material mechanics and blood parameters of the steel cylinder are: $\rho=7.85 \mathrm{~g} / \mathrm{cm}^{3}$, $E=210 \mathrm{GPa}, v=0.32, \sigma_{y}=0.355 \mathrm{GPa}, c_{V}=468.2 \mathrm{~J} /(\mathrm{kg} \cdot \mathrm{K})$.

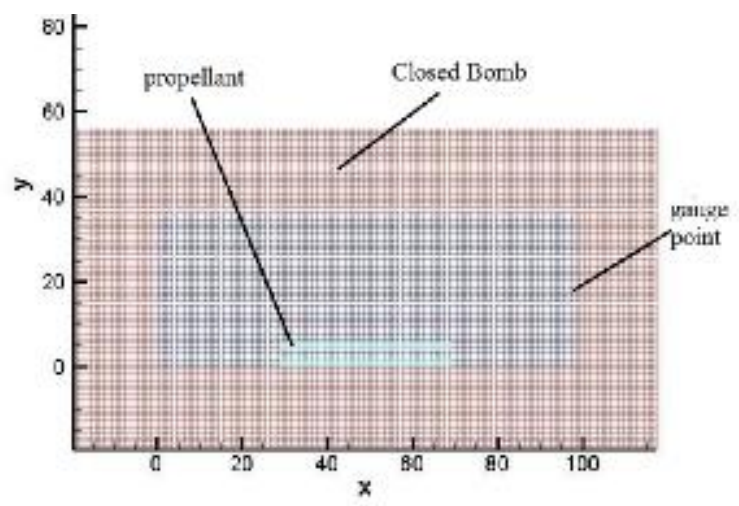

Fig.2 Numerical model schematic diagram of MPM

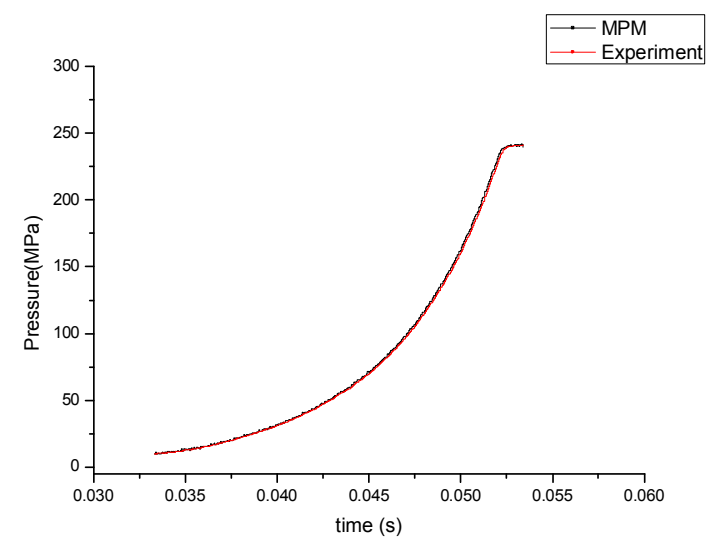

Fig.3 The curve of pressure-time at the point

Figure 3 shows the pressure time curve at the measuring point. It can be seen from the figure that the MPM numerical analysis agrees well with the experimental data. With the reaction of the propellant, 
the pressure at the measuring point increases, About $240 \mathrm{MPa}$ to reach the maximum, the reaction is over, the cylinder pressure to maintain balance.

Figure 4 (a) for the burning rate - pressure curve. It can be seen from the figure that the MPM numerical analysis agrees well with the experimental data, and is consistent with the burning rate pressure formula $u=u_{1} P^{n}$ : where: $u$ for the burning rate, $u_{1}$ the burning rate coefficient $n$ is the pressure index. The experimental data show that the combustion rate is gradually reduced to 0 as the reaction reaches the end, and is consistent with the actual situation. The MPM numerical analysis data can be used to reflect the change of burning rate with pressure, which is in accordance with the basic formula of burning rate. On the burning rate - pressure formula for natural logarithm: $\ln u=\ln u_{1}+n \ln P$,Figure 4.3 (b) $\ln u-\ln P$ curve, the slope is the pressure index $n$. Thus, $n=\frac{\mathrm{d} \ln u}{\mathrm{~d} \ln p}=\frac{\ln u_{1}-\ln u_{2}}{\ln p_{1}-\ln p_{2}}, n-P$ curve as shown in Figure 4.3 (c), the range $n$ is $0.80197-0.80244$.

From the burning rate formula can get $u_{1}=\exp (\ln u+n \ln P)$, the curve $u_{1}-P$ is shown in Figure 4.3 (d), the range $u_{1}$ is $0.19195-0.19240$. The burning rate $u_{1}$ and pressure index $n$ measured in the experiment are shown in Table 4.1. It can be seen that the MPM numerical analysis is in good agreement with the experimental data.

Table $1 \quad u_{1}$ and $\mathrm{n}$ of MPM and experiment

\begin{tabular}{ccc}
\hline & MPM & experiment \\
\hline$u_{1}(\mathrm{~cm} /(s \cdot M P a))$ & $0.19195-0.19240$ & 0.1922 \\
$\mathrm{n}$ & $0.80197-0.80244$ & 0.8022 \\
\hline
\end{tabular}

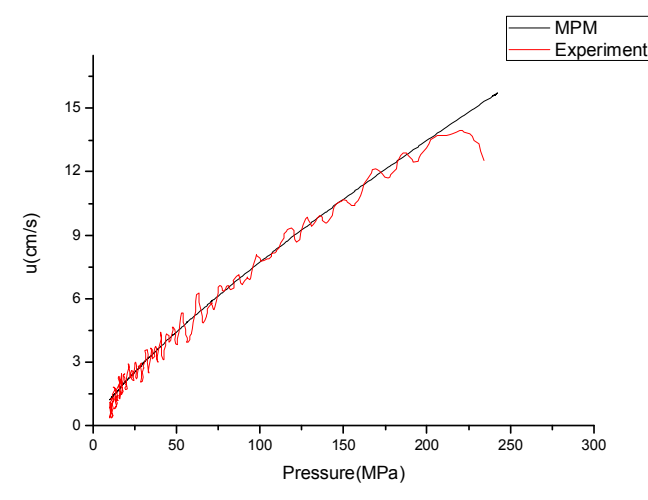

(a) The curve of $u-P$

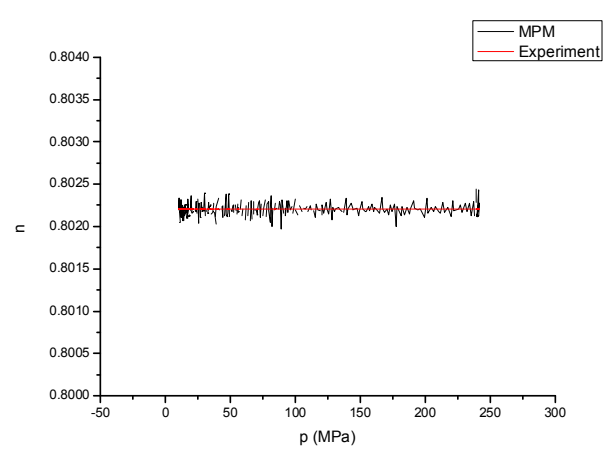

(c) The curve of n-p

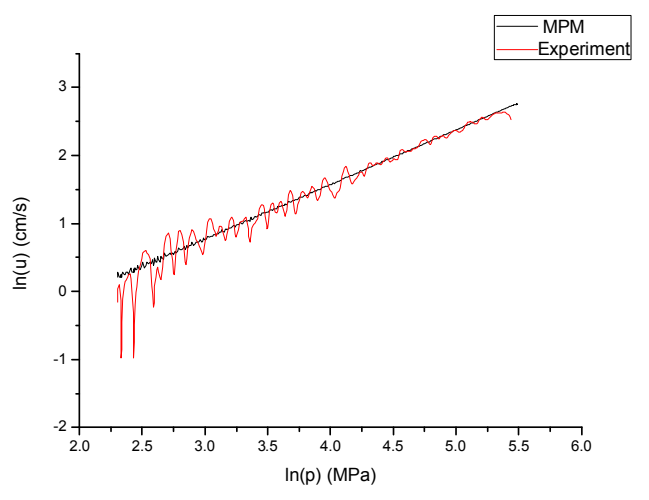

(b) The curve of lnu-lnp

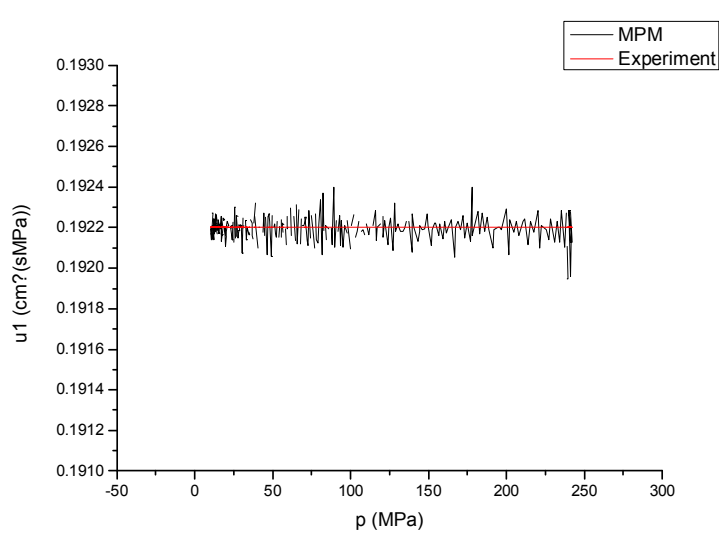

(d) The curve of $u 1-p$

Fig4 The characteristic curve of propellant combustion 


\section{Conclusion}

The material point method inherits the advantages of the Lagrangian and Eulerian methods, and has great advantages in dealing with large deformation and multi-media coupling problems. On the basis of material point method, the burning problem of energetic materials was studied, and the combustion model of energetic materials based on substance point method was established. A method of substance point method for the burning of energetic materials was prepared by FORTRAN language. Point method is extended to the field of combustion numerical calculation, and the numerical simulation of the closed-burst test is carried out. The combustion characteristic curves of the pressure time-history curve and the burning-pressure curve of the energetic materials are obtained. At the same time, the experimental data and the burning rate - the pressure formula is compared and the results are in good agreement, which provides a new way for the combustion simulation of the energetic materials, and provides a numerical method and important reference for the study of the combustion characteristics of the energetic materials

\section{Acknowledgements}

This work was financially supported by the Key Laboratory of Shock Wave Physics and Detonation Physics Open fund (1sdkfjj-2014-0002)

\section{References}

[1] Rui Xiao-ting, FENG Bin-bin, WANG Yan, et al. Research on Evaluation Method for Launch Safety of Propellant Charge[J]. ACTA ARMAMENTARII, 2015, 1000-1093(2015)01-0001-11.In Chinese

[2] WEN Gang, DU Ping, LIAO Xin. Principle and Method of Measuring Actual Burning Rate of Propellant by Closed Bomb[J]. Chinese Journal of Explosives \& Propellants, 2011, 34(3):57-60. In Chinese

[3] CHEN Tao, RUI Xiao-ting, HONG Jun, et al. Semi-closed Bomb Simulation Using Study Dynamic Extrusion Stress of Charge Bed[J]. Journal of System Simulation, 2007,19(17):4075-4078. In Chinese

[4] LI GUANG-yao, Kalilu. Element Free Galerkin Method for Elasto-Plastic Problems with Large Deformation Distortion. Journal of Hunan University, 2003,30(1):47-19. In Chinese

[5] Bardenhagen S G. The Material Point Method and Simulation of Wave Propagation in Heterogeneous Media[J]. AIP Conference Proceedings, 2004, 706:P187-192.

[6] CHEN Chen, LIU Gui-e, JIANG Jin-yong, et al.Experimental Research on the Thermal Safety of SF-3 Gun Propellant[J]. Chinese Journal of Explosives \& Propellants, 2014,37(3)70-73. In Chinese

[7] Sun Bao-ping, Duan Zhuo-ping, Zhang Hai-ying, et al. Experiment and numerical simulation on ignition of charge by fragment impact[J]. EXPLOSION AND SHOCK WAVE, 2013,33(5):456-462. In Chinese 